\title{
Research Square \\ Estimation of renal perfusion based on measurement of Rubidium-82 clearance by PET/CT scanning in healthy subjects
}

Stine Sundgaard Langaa ( $\sim$ stinlg@rm.dk)

University Clinic in Nephrology and Hypertension https://orcid.org/0000-0003-4525-7283

Thomas Guldager Lauridsen

University Clinic in Nephrology and Hypertension, Department of Medical Research, Gødstrup Hospital, lægaardvej 12J, 7500 Holstebro, Denmark

\section{Frank Holden Mose}

University Clinic in Nephrology and Hypertension, Department of Medicical Research, Gødstrup Hospital, Lægaardvej 12J, 7500 Holstebro, Denmark

\section{Claire Anne Fynbo}

Department of Nuclear Medicine, Gødstrup Hospital, Denmark

\section{Jørn Theil}

Department of Nuclear Medicine, Gødstrup Hospital, Denmark

\section{Jesper Nørgaard Bech}

University Clinic in Nephrology and Hypertension, Department of Medical Research, Gødstrup Hospital, Lægaardvej 12J, 7500 Holstebro, Denmark

\section{Original research}

Keywords: PET/CT, Rubidium-82, Pharmacokinetic modelling, Renal blood flow, Effective renal plasma flow

Posted Date: February 7th, 2021

DOl: https://doi.org/10.21203/rs.3.rs-87482/v2

License: (c) (i) This work is licensed under a Creative Commons Attribution 4.0 International License.

Read Full License 


\section{Estimation of renal perfusion based on measurement of Rubidium-82}

\section{clearance by PET/CT scanning in healthy subjects}

Stine Sundgaard Langaa ${ }^{1}$, Thomas Guldager Lauridsen ${ }^{1}$, Frank Holden Mose ${ }^{1}$, Claire Anne

$$
\text { Fynbo }^{2}, \text { Jørn Theil }{ }^{2,3} \text { and Jesper Nørgaard Bech }{ }^{1}
$$

\footnotetext{
${ }^{1}$ University Clinic in Nephrology and Hypertension, Gødstrup Hospital and Aarhus University, Denmark and ${ }^{2}$ Department of Nuclear Medicine, Gødstrup Hospital, Denmark and ${ }^{3}$ Department of Clinical Medicine, Aarhus University
}

\section{Corresponding author:}

Stine Sundgaard Langaa

University Clinic in Nephrology and Hypertension

Department of Medical Research

Gødstrup Hospital

Lægaardvej 12J

7500 Holstebro

Denmark

$+4578436587$

stinlg@rm.dk 


\section{Abstract}

2 Background: Changes in renal blood flow (RBF) may play a pathophysiological role in

3 hypertension and kidney disease. However, RBF determination in humans has proven difficult. We

4 aimed to confirm the feasibility of RBF estimation based on positron emission tomography/ computed tomography (PET/CT) and rubidium-82 $\left({ }^{82} \mathrm{Rb}\right)$ using the abdominal aorta as input

6 function in a 1-tissue compartment model.

7 Methods: Eighteen healthy subjects underwent two dynamic ${ }^{82} \mathrm{Rb}$ PET/CT scans in two different

8 fields of view (FOV). FOV-A included the left ventricular blood pool (LVBP), the abdominal aorta

9 (AA) and the majority of the kidneys. FOV-B included AA and the kidneys in their entirety. In

10 FOV-A, an input function was derived from LVBP and from AA; in FOV-B from AA. 1-tissue

11 compartmental modeling was performed using tissue time activity curves generated from volumes

12 of interest contouring the kidneys, where the renal clearance of ${ }^{82} \mathrm{Rb}$ is represented by the $\mathrm{K}_{1}$ kinetic

13 parameter. To investigate the correct interpretation of $\mathrm{K}_{1}$, we assumed to first estimate effective

14 renal plasma flow (ERPF) by extrapolating clearance values $\left(\mathrm{ml} / \mathrm{min} / \mathrm{cm}^{3}\right)$ to whole kidney values

15 ( $\mathrm{ml} / \mathrm{min}$ ) using the estimated total kidney volume. Thereafter, RPF was estimated from ERPF using

16 an assumed extraction fraction (0.89). Lastly, RBF was estimated from RPF using measured

17 haematocrit values. Intra-assay coefficients of variation and inter-observer variation were

18 calculated.

19 Results: For both kidneys, $\mathrm{K}_{1}$ values derived from AA did not differ significantly from values

20 obtained from LVBP, neither were significant differences seen between AA in FOV-A and AA in

21 FOV-B, nor between the right and left kidneys. For both kidneys, the intra-assay coefficients of

22 variation were low $(\sim 5 \%)$ for both input functions. The measured $\mathrm{K}_{1}$ of $2.80 \mathrm{ml} / \mathrm{min} / \mathrm{cm}^{3}$ suggests,

23 for young healthy subjects, an estimated total renal perfusion normalized to body surface area of

$24860 \pm 129 \mathrm{ml} / \mathrm{min} / 1.73 \mathrm{~m}^{2}$ and subsequently an estimated RBF of $1494 \pm 221 \mathrm{ml} / \mathrm{min} / 1.73 \mathrm{~m}{ }^{2}$. 
1 Conclusion: RBF estimation based on PET/CT and ${ }^{82} \mathrm{Rb}$ using AA as input function in a 1-tissue

2 compartment model is feasible in a single FOV. The measured $\mathrm{K}_{1}$ clearance values are most likely representative of ERPF rather than estimated RBF values.

Keywords:

PET/CT, Rubidium-82, Pharmacokinetic modelling, Renal blood flow, Effective renal plasma flow

\section{Background}

8 Kidney disease and hypertension are major contributors to the overall global disease burden. In the

9 pathogenesis of acute kidney injury (AKI), renal ischemia, as a result of a reduction in total RBF,

10 has been accepted as a significant factor. However, recent studies suggest that renal hypoperfusion

11 may play a less important role $(1,2)$. In fact, RBF measurements in sepsis-associated AKI have

12 shown much discrepancy; reduced, normal, or even increased RBF have been reported (3-5). In

13 patients with chronic kidney disease (CKD), RBF is reduced compared with controls $(6,7)$,

14 possibly contributing to the progression of renal dysfunction. In renal circulation studies, most

15 patients with essential hypertension display reduced $\operatorname{RBF}(8,9)$; the greatest reduction demonstrated

16 in malignant hypertension $(9,10)$. Additionally, renal vasoconstriction has been identified in pre-

17 hypertensive adults, indicating that renal vascular abnormalities could be a cause of hypertension

18 rather than caused by hypertension $(11,12)$.

19 Quantification of renal perfusion in humans has proven difficult. Clearance-based methods estimating ERPF are time consuming and burdensome for patients (13-15) and alternative

21 radiological imaging techniques assessing $\mathrm{RBF}$, such as magnetic resonance imaging and 22 ultrasonography, all have considerable limitations $(7,16)$ - none of which have been routinely 23 implemented in clinical practise. Dynamic positron emission tomography (PET) using perfusion 24 tracers is currently considered the most accurate, non-invasive method for determination of organ 
1 perfusion. Thus, with good homogeneity and high perfusion rate, the kidneys are well suited for

2 PET studies.

3 PET scans using ${ }^{82} \mathrm{Rb}$ are routinely performed to assess myocardial blood flow in patients suspected

4 of ischemic heart disease $(17,18) .{ }^{82} \mathrm{Rb}$ is a potassium analogue with a short half-life of 75 seconds,

5 produced in a generator by the radioactive decay of strontium- $82\left({ }^{82} \mathrm{Sr}\right)$. Due to its high first-pass

6 renal extraction $(\sim 90 \%)$ and slow wash-out, ${ }^{82} \mathrm{Rb}$ is well suited for mathematic modelling of RBF

7 using dynamic PET-methods (19).

8 The first human ${ }^{82} \mathrm{Rb}$ PET/CT study of renal perfusion showed high image quality, resolution and

9 contrast, as well as demonstrated a high natural ${ }^{82} \mathrm{Rb}$ renal uptake $(20,21)$. RBF was evaluated

10 using a 1-tissue compartment model, where the $\mathrm{K}_{1}$ parameter is presumed to represent estimated

11 RBF.

12 Compartmental modelling requires an input function (IF) described by a blood-pool time activity

13 curve. In quantification of myocardial blood flow, the LVBP has been validated as an image-

14 derived input function (IDIF) $(22,23)$, obviating the need for arterial blood sampling. However, the

15 LVBP is not necessarily ideal for studying renal perfusion, as the LVBP and the kidneys in their

16 entirety may not fit within a single limited axial scanner-FOV. This is especially true for older PET-

17 scanners. In order, to ensure that an IF is estimated as correctly as possible, as well as to minimize

18 radiation dose associated with the scanning, inclusion of the blood-pool and the kidneys in their

19 entirety in the same FOV, is important. This can be accomplished if the AA can replace the LVBP

20 as IF in the model, as suggested by Tahari et al. (21).

21 To determine whether this method is suitable for clinical, reliable assessment of RBF, this study

22 further investigates the substitution of AA as a valid alternative to LVBP by comparing the

23 resulting $\mathrm{K}_{1}$ values obtained from use of the two different IFs in a substantially larger study

24 population than that of (21). We also evaluate method precision by determination of intra-assay

25 coefficients of variation for both IFs as well as assess inter-observer variation. Furthermore, 
1 existing literature assumes that the perfusion quantity measured using ${ }^{82} \mathrm{Rb}$ is estimated $\mathrm{RBF}(21$,

2 24). Early investigations into the exchange rates of radioactive potassium and rubidium between

3 plasma and erythrocytes showed rates of $\sim 2 \%$ per hour $(25,26)$, implying that initially, and hence

4 during renal uptake studies, the majority of injected ${ }^{82} \mathrm{Rb}$ will be almost exclusively present in

5 plasma. We discuss and question whether flow values measured by ${ }^{82} \mathrm{Rb}$ clearance are actually

6 representative of RBF, or whether they should be interpreted as estimates of ERPF.

\section{Methods}

\section{$9 \quad$ Study design}

10 This study was performed as a randomized cross-over study (Fig. 1). During a period of

11 approximately 45 minutes, each subject underwent four 8-minute dynamic ${ }^{82} \mathrm{Rb} \mathrm{PET} / \mathrm{CT}$ scans in

12 two different bed positions, A and B (FOV-A and FOV-B). In each bed position, duplicate scans

13 were performed.

\section{Participants}

15 Healthy participants were recruited through advertisement, primarily at local educational

16 institutions. Prior to inclusion, each participant completed a screening program. Screening consisted

17 of a medical history; a clinical examination including measurements of weight, height, and blood

18 pressure; electrocardiography as well as blood tests to determine electrolytes, creatinine, albumin,

19 alanine aminotransferase (ALAT), leucocytes, haemoglobin, haematocrit and thrombocytes. Urine

20 was screened for leucocytes, glucose, nitrite, ketones, and haemoglobin. Inclusion criteria were:

21 men and women aged 18-40 years with a body mass index (BMI) in the range $18.5-30.0 \mathrm{~kg} / \mathrm{m}^{2}$.

22 Exclusion criteria were: medical treatment (except hormonal contraceptives); pregnancy or

23 breastfeeding; smoking; substance abuse; alcohol consumption $>14$ units ${ }^{1}$ per week for men and $>7$

\footnotetext{
${ }^{1}$ Danish alcohol unit $=15 \mathrm{ml}(12 \mathrm{~g})$ pure alcohol.
} 
1 units per week for women; signs of clinically relevant kidney disease, heart disease, liver disease or

2 endocrine disease in the history, clinical examination, or the paraclinical tests; hypertension;

3 neoplastic disease, and blood donation within 1 month of the examination day. Withdrawal criteria

4 were development of exclusion criteria or withdrawal of consent.

\section{$5 \quad$ Number of subjects}

617 subjects are required to detect a $0.40 \mathrm{ml} / \mathrm{min} / \mathrm{cm}^{3}$ difference in RBF (standard deviation (SD)

$7 \quad 0.38 \mathrm{ml} / \mathrm{min} / \mathrm{cm}^{3}$ ) for a $5 \%$ significance level and power of $80 \%$. To allow for dropout, 20 subjects

8 were included.

\section{Pre-scan procedure}

10 For 24 hours preceding the acquisition of PET/CT scans, fluid intake was standardized to $35 \mathrm{ml} / \mathrm{kg}$

11 body weight of still water, subjects maintained a free diet and were instructed to avoid strenuous

12 exercise. Subjects arrived at 8 a.m. at the Department of Nuclear Medicine, Herning Hospital,

13 Regional Hospital West Jutland, Denmark after an overnight fast. In female subjects, pregnancy

14 was ruled out.

\section{Radiopharmaceutical}

16 On each day of examination, the ${ }^{82} \mathrm{Sr} /{ }^{82} \mathrm{Rb}$ generator (Cardiogen-82; Bracco Diagnostics Inc.,

17 Monroe Township, NJ, USA) was quality checked according to approved guidelines (Bracco

18 Diagnostics Inc.) including test for breakthrough of ${ }^{82} \mathrm{Sr} /{ }^{85} \mathrm{Sr}$. The generator was calibrated to

19 deliver a dose of $555 \mathrm{MBq}(15 \mathrm{mCi}){ }^{82} \mathrm{Rb}$ for each injection, which was administered automatically

20 using a pre-programmed pump and infusion system. The subjects received four doses in total.

\section{$21 \quad$ PET/CT scanning}

22 All PET/CT scans were performed on the same scanner (Siemens Biograph mCT; 64 slice-4R) with

23 a $22 \mathrm{~cm}$ axial FOV. On each day of examination, the PET/CT scanner was quality checked and

24 calibrated according to system required procedures. A peripheral venous catheter (Venflon) was

25 placed in a cubital vein for ${ }^{82} \mathrm{Rb}$ injection. Subjects rested in a sitting position for approximately 30 
1 minutes before voiding. They were then placed in a supine position in the PET/CT scanner with

2 arms extended above the head and the generator infusion system connected to the Venflon. All

3 subjects underwent two consecutive duplicate PET/CT scans. The duplicate scans were acquired in

4 bed position A (FOV A), including the LVBP, the AA and as much of the kidneys as possible (Fig.

5 2a) and bed position B (FOV B), including the AA and the kidneys in their entirety (Fig. 2b).

6 Computer generated randomization determined the acquisition sequence for the two FOVs for each

7 participant. In each bed position, an initial planar scout image was acquired to determine

8 positioning of the scanner over the required FOV. Following positioning, a low-dose CT scan was

9 performed immediately followed by a bolus injection of $555 \mathrm{MBq}{ }^{82} \mathrm{Rb}$ and a dynamic PET-scan in

10 list-mode for 8 minutes synchronized with the start of injection (21). Sequentially, and 10 minutes

11 after the first PET scan was initiated, a second dose of ${ }^{82} \mathrm{Rb}$ was administered and a duplicate PET

12 scan performed in list-mode for 8 minutes. The bed position was then shifted, and the procedure

13 repeated for the second FOV.

14 Low-dose CT scans (25 mAs, $100 \mathrm{kV})$ were performed for attenuation correction purposes only.

15 PET data were acquired in dynamic list-mode, which was re-binned using 32 frames $(20 \times 6 \mathrm{~s}, 5 \times 12$

$16 \mathrm{~s}, 4 \times 30 \mathrm{~s}$ and $3 \times 60 \mathrm{~s}$ ) and iteratively reconstructed ( 21 subsets, 2 iterations) using Siemens TrueX

17 and time-of-flight reconstruction in a matrix of $128 \times 128$ (voxel size: $6.4 \times 6.4 \times 3.0 \mathrm{~mm}^{3}$ ) and post-

18 filtered with a $5.0 \mathrm{~mm}$ Gaussian filter to produce attenuation and decay corrected dynamic

19 sequences. We found it unnecessary to correct for motion of the kidneys.

20 The effective radiation dose associated with the study was $<4$ millisieverts (mSv): each low-dose

$21 \mathrm{CT}$ scan contributed $0.4 \mathrm{mSv}$ and each $555 \mathrm{MBq}$ bolus injection of ${ }^{82} \mathrm{Rb}$ contributed with 1.26

$22 \mu \mathrm{Sv} / \mathrm{MBq}(20)$.

\section{Analysis of ${ }^{\mathbf{8 2}} \mathrm{Rb}$ PET/CT studies}

24 A 1-tissue compartment model was used for flow estimation (21), as illustrated in Fig. 3. The $\mathrm{K}_{1}$

25 parameter represents the renal clearance of ${ }^{82} \mathrm{Rb}$, where $\mathrm{K}_{1}\left(\mathrm{ml} / \mathrm{min} / \mathrm{cm}^{3}\right)$ is equal to the product of 
1 the blood flow component carrying the ${ }^{82} \mathrm{Rb}$ (erythrocyte and/or plasma) and its extraction fraction

2 (EF) in the kidneys. Due to ${ }^{82} \mathrm{Rb}$ having a high first pass extraction ( $\left.90 \%\right)(19)$, its uptake rate $\mathrm{K}_{1}$

3 will be closely related to, and hence can be used as, an estimate of flow (27). Compartmental

4 modelling was performed using the PMOD software (PMOD Technologies Ltd., Zurich,

5 Switzerland, version 4.01).

6 Time-activity-curves (TACs) were obtained by defining relevant volumes of interest (VOIs) in the

7 various anatomical regions-of-interest (Fig. 4), with the LVBP and AA defining IFs for the kinetic

8 modelling. All TACs were obtained as the mean activity concentrations measured in the VOIs. The

9 LVBP was defined in FOV-A using a limiting box and the hot-contour tool with a typical cut-off 45

$10-60 \%$ of the maximum limiting box activity. Ensuring avoidance of surrounding activity in the

11 right and left ventricular luminae, a background VOI was manually placed centrally in the left

12 ventricular wall and defined on at least 10 contiguous slices. Partial-volume effect (PVE) and spill-

13 over activity from the left ventricular wall was corrected adopting the method described by Katoh et

14 al. (28):

$$
R_{A}(t)=\beta \cdot C_{A}(t)+(1-\beta) \cdot \rho \cdot C_{B g}(t)
$$

16 where $\mathrm{C}_{\mathrm{A}}(\mathrm{t})$ represents the corrected LVBP activity, $\mathrm{R}_{\mathrm{A}}(\mathrm{t})$ is the measured LVBP activity, $\mathrm{C}_{\mathrm{Bg}}(\mathrm{t})$ the 17 measured myocardial activity, $\rho$ the partition coefficient of water in the myocardium (0.91) and $\beta$

18 the recovery coefficient required to correct measured image activity concentration values to the

19 correct activity present in the LVBP. Calibration measurement using the NEMA-IQ phantom filled

20 with a background to hotspot ratio $1: 10$, known ${ }^{82} \mathrm{Rb}$ activity concentrations and the same

21 reconstruction parameters as for the study, determined $\beta$ to be 0.71 for the LV.

22 The AA-VOI was defined in both FOVs using a box $\left(10 \times 10 \times 30 \mathrm{~mm}^{3}\right)$ placed in the lumen of the 23 abdominal aorta cranially to the departure of the renal arteries. An aortic background VOI was 
1 defined within a limiting box around the AA-VOI by applying a cold-contour with typical cut-off of

$24-5 \%$ of the maximum activity and excluding all structures not representing background activity.

3 Based on the formulation for PVE and spill-over correction for the LVBP of (28), the measured AA

4 activity concentration can be similarly corrected for possible background and PVE contributions

5 using:

$$
R_{A}(t)=\beta \cdot C_{A}(t)+(1-\beta) \cdot C_{B g}(t)
$$

7 where $\mathrm{C}_{\mathrm{A}}(\mathrm{t})$ represents the corrected $\mathrm{AA}$ activity, $\mathrm{R}_{\mathrm{A}}(\mathrm{t})$ is the measured $\mathrm{AA}$ activity, $\mathrm{C}_{\mathrm{Bg}}(\mathrm{t})$ is the

8 measured aortic background activity and $\beta$ the necessary recovery coefficient related to the AA

9 geometry and analysis VOI-placement. Measurement of known ${ }^{82} \mathrm{Rb}$ activity concentrations in a

10 homogeneous home-made phantom simulating the AA/background geometry, determined $\beta$ to be

110.612.

12 Tissue-TACs for both kidneys were obtained using hot-contouring in both FOVs as described

13 previously and $\mathrm{K}_{1}$ values for each kidney obtained for both LVBP and AA IFs using the 1-tissue

14 compartment model. A blood volume fraction of 10\%, applied as a fixed parameter in the PMOD

15 kinetic modelling, was used to account for activity from the fractional blood volume within the

16 VOIs contouring the kidneys (29). Additionally, a recovery coefficient $\beta=0.643-$ measured in a

17 large, homogeneous phantom volume - was applied to the kidney-TAC data. Due to large kidney

18 volumes and careful placement of applied VOIs at a distance from the kidney boundary walls,

19 effects from PVE and spill-over are negligible and do not need correction. However, as the Siemens

20 system software does not itself correct for ${ }^{82} \mathrm{Rb}$ count efficiency, manual correction for this in the

21 kidney data is also essential to ensure that the measured activity concentrations in the different

22 organ VOIs, when corrected for all necessary scanner and reconstruction effects, are relatively

23 correct to each other. 
$1 \quad \mathrm{As}^{82} \mathrm{Rb}$ is a small molecule, it will be distributed in the water phase in plasma and as such is freely

2 diffusible in the interstitium and constitutes the extravascular background. This intercellular

3 distribution is included in the input function, which is modelled using a 3-exponential model in

$4 \quad$ PMOD with the background activity corrected using the $\beta$-correction procedure.

5 Kinetic analysis was performed independently by two observers: a medical resident (observer 1)

6 and an experienced nuclear medical physician (observer 2).

\section{$7 \quad$ Renal blood flow estimation}

8 Assuming that, after intravenous injection and throughout the 8-minute duration of the study

9 acquisition ${ }^{82} \mathrm{Rb}$ is almost exclusively distributed in the plasma $(25,26)$, then ERPF can be

10 estimated using the measured ${ }^{82} \mathrm{Rb}$ clearance $\left(\mathrm{K}_{1}\right)$ and the total kidney volume $\left(\mathrm{V}_{\text {Total }}\right)$ as

11 determined by the renal contour volumes described above:

$$
E R P F=K_{1} \cdot V_{\text {Total }}
$$

13 RPF can be estimated using the assumed EF $(\sim 0.89(19))$ :

$$
R P F=\frac{E R P F}{E F}
$$

and subsequently, estimated RBF can be calculated from estimated RPF using the haematocrit (Hct)

16 as:

$$
R B F=\frac{R P F}{1-H c t}
$$

18 Estimated RPF and RBF results are presented normalized to body surface area (BSA) using the

19 Dubois formula:

$$
B S A=0.007184 \cdot \text { height }^{0.725} \cdot \text { weight }^{0.425}
$$

21 where quantity units are given in: BSA $\left[\mathrm{m}^{2}\right]$, height $[\mathrm{cm}]$ and weight $[\mathrm{kg}]$. 


\section{Statistical analysis}

2 Statistical tests were performed using SPSS Statistics ver. 20 (IBM Corp., Armonk, NY, USA). For

3 each subject, the result for $\mathrm{K}_{1}$ was defined as the mean value of the two independent $\mathrm{K}_{1}$ values

4 determined for each FOV for both input functions.

5 Values are presented as mean \pm SD for all completing subjects. Paired sample t-testing was used for

6 comparison of $\mathrm{K}_{1}$ values obtained using LVBP and AA IFs, where $\mathrm{p}<0.05$ was considered

7 statistically significant.

8 Intra-assay coefficients of variation were calculated for each kidney based on the duplicate $\mathrm{K}_{1}$

9 determinations in each FOV. Inter-observer variability was assessed using the intra-class correlation

10 coefficient (ICC) with 95\% confidence interval (CI) (30).

\section{Results}

\section{Demographics}

14 The participation flow chart for the study is depicted in Fig. 5. Eighteen healthy subjects completed

15 the study and had scans accepted for analysis. Clinical and biochemical characteristics are shown in 16 Table 1.

Table 1 Clinical and biochemical characteristics $(n=18)$

\begin{tabular}{lc}
\hline Age (years) & $21 \pm 4$ \\
Gender (women/men) & $7 / 11$ \\
BMI $\left(\mathrm{kg} / \mathrm{m}^{2}\right)$ & $24.1 \pm 2.5$ \\
Office SBP (mmHg) & $127 \pm 9$ \\
Office DBP (mmHg) & $73 \pm 10$ \\
Heart rate (beats/min) & $71 \pm 11$ \\
P-alanine aminotransferase (U/L) & $26 \pm 11$ \\
P-sodium (mmol/L) & $140 \pm 2$ \\
P-potassium (mmol/L) & $3.7 \pm 0.2$ \\
P-albumin (g/L) & $43 \pm 3$
\end{tabular}




$\begin{array}{lc}\text { P-creatinine }(\mu \mathrm{mol} / \mathrm{L}) & 73 \pm 13 \\ \text { eGFRMdRD }\left(\mathrm{mL} / \mathrm{min} / 1.73 \mathrm{~m}^{2}\right) & 118 \pm 11 \\ \text { B-hemoglobin }(\mathrm{mmol} / \mathrm{L}) & 9.0 \pm 0.4 \\ \text { B-leucocytes }\left(\times 10^{9} / \mathrm{L}\right) & 7.1 \pm 1.9 \\ \text { B-thrombocytes }\left(\times 10^{9} / \mathrm{L}\right) & 275 \pm 48 \\ \text { B-haematocrit } & 0.42 \pm 0.02\end{array}$

Data are presented as mean \pm SD. BMI, Body Mass Index; SBP, systolic blood pressure; DBT, diastolic blood pressure; eGFR $_{\text {MDRD }}$, estimated glomerular filtration rate calculated using the Modification of diet in renal Disease Study equation; EVF, erythrocyte volume fraction

\section{Input curves}

6 Fig. 6 illustrates typical TACs generated from VOIs in the LVBP and the myocardium, as well as $\beta$ 7 corrected activity in LVBP. For all curves, the activity peaks rapidly. However, whereas for LVBP

8 the flow-peak is followed by a continuous decline, the myocardial activity plateaus around 1.0

9 minute post injection (p.i.) after the decline of the initial flow-peak.

10 Similarly, Fig. 7 illustrates typical TACs generated from VOIs placed in the AA and the aortic

11 background as well as the $\beta$-corrected AA activity. As for LVBP, AA activities rapidly reach their 12 maximum peak followed by rapid declines, while the aortic background activity rises slowly until

13 reaching a plateau between 0.5 and 3.0 minutes p.i. followed by a slow decline.

14 Fig. 8 shows a typical example of the relative ${ }^{82} \mathrm{Rb}$ activity concentrations (corrected TAC data)

15 between the organ VOIs of the LVBP, AA and kidneys. The injected bolus peaks for the LVBP and

16 AA are very similar, reaching nearly the same maximum peak values in the same time-bin after

17 injection, whereas, the kidney uptake rises more slowly until reaching a plateau between 1.5 and 4.5 18 minutes p.i. followed by a slow decline.

\section{Renal clearance - measurement of $K_{1}$, estimation of ERPF and RBF}

20 High renal uptake of ${ }^{82} \mathrm{Rb}$ was demonstrated with no discernible urinary activity (Fig. 9). 
1 The caudal part of the kidneys was outside FOV-A in 5 out of the 18 completing subjects.

2 Measurements in FOV-B showed that $\mathrm{K}_{1}$ for the excluded caudal sections did not differ from the

3 global $\mathrm{K}_{1}$ for the kidneys.

4 Table 2 presents the mean $\mathrm{K}_{1}$ results for all IFs applied in the analysis. $\mathrm{K}_{1}$ values using the AA IF

5 were not significantly different from those using LVBP. No significant difference was observed

6 between left and right kidneys.

Table 2 Mean $\mathrm{K}_{1}$ values for the investigated input functions

\begin{tabular}{|c|c|c|c|}
\hline & LVBP & $\mathrm{AA}(\mathrm{FOV}-\mathrm{A})$ & $\mathrm{AA}(\mathrm{FOV}-\mathrm{B})$ \\
\hline Right kidney & $2.75 \pm 0.42$ & $2.86 \pm 0.48^{*}$ & $2.82 \pm 0.45^{\wedge}$ \\
\hline Left kidney & $2.71 \pm 0.42$ & $2.82 \pm 0.45^{*}$ & $2.79 \pm 0.42^{\wedge}$ \\
\hline
\end{tabular}

Intra-assay coefficients of variation for the duplicate VOIs for each FOV were calculated for K1

13 derived from LVBP and AA IFs. As illustrated in Table 3, the intra-assay coefficients of variation 14 were similar $(\sim 5 \%)$ for both IFs, with those for AA being slightly lower than those for LVBP IFs.

15

16

17

Table 3 Intra-assay coefficients of variation

\begin{tabular}{lccc} 
& LVBP & AA (FOV-A) & AA (FOV-B) \\
\hline Right kidney & 5.6 & 4.4 & 4.4
\end{tabular}

\begin{tabular}{llll} 
Left kidney & 5.7 & 4.3 & 4.4 \\
\hline
\end{tabular}

18

Data are presented as percentages. LVBP, left ventricular blood pool; AA, abdominal aorta; FOV-A, field of view A; FOV-B, field of view B..

21 Table 4 shows inter-observer variability when using LVBP and AA IFs for the two FOVs. Using

22 LVBP, ICC was indicative of good to excellent reliability for both kidneys. For AA, ICC was

23 suggestive of excellent reliability for both kidneys in FOV-A and FOV-B. 
Table 4 Inter-observer variability

\begin{tabular}{lccc}
\hline & LVBP & AA (FOV-A $)$ & AA (FOV-B) \\
\hline Right kidney & $0.874(0.696 ; 0.951)$ & $0.971(0.925 ; 0.989)$ & $0.969(0.920 ; 0.988)$ \\
& & & \\
Left kidney & $0.880(0.708 ; 0.953)$ & $0.972(0.926 ; 0.989)$ & $0.965(0.909 ; 0.987)$ \\
\hline
\end{tabular}

3 Data are presented as ICC estimates with $95 \%$ confidence intervals in parentheses.

4 LVBP, left ventricular blood pool; AA, abdominal aorta; FOV-A, field of view A; FOV-B, field of view B.

Under the assumptions described in Methods and using equations 3-6, total flow values were estimated (Table 5). Total ERPF, RPF and RBF were estimated to be $766 \pm 114 \mathrm{ml} / \mathrm{min} / 1.73 \mathrm{~m}^{2}$, $860 \pm 129 \mathrm{ml} / \mathrm{min} / 1.73 \mathrm{~m}^{2}$ and $1494 \pm 221 \mathrm{ml} / \mathrm{min} / 1.73 \mathrm{~m}^{2}$ respectively.

Table 5 Estimation of ERPF and RBF based on ${ }^{82} \mathrm{Rb}$ clearance values $\left(\mathrm{K}_{1}\right)$ using AA activity in 12 FOV-B

\begin{tabular}{cccccc}
\hline $\begin{array}{c}\text { Average } \mathrm{K}_{1} \\
\left(\mathrm{ml} / \mathrm{min} / \mathrm{cm}^{3}\right)\end{array}$ & $\begin{array}{c}\text { Total renal volume } \\
\left(\mathrm{cm}^{3}\right)\end{array}$ & $\begin{array}{c}\text { Total ERPF } \\
(\mathrm{ml} / \mathrm{min})\end{array}$ & $\begin{array}{c}\text { Total RPF } \\
(\mathrm{ml} / \mathrm{min})\end{array}$ & $\begin{array}{c}\text { Average } \\
\text { Hct }\end{array}$ & $\begin{array}{c}\text { Total RBF } \\
(\mathrm{ml} / \mathrm{min})\end{array}$ \\
\hline $2.80 \pm 0.43$ & $296 \pm 30$ & $825 \pm 122$ & $927 \pm 138$ & $0.42 \pm 0.02$ & $1612 \pm 248$
\end{tabular}

Data are presented as means \pm SD. Total ERPF is calculated as the product of $K_{1}$ and total kidney volume. Total RPF is estimated from ERPF using an assumed EF of 0.89 (19). RBF is estimated from RPF and the measured Hct. ERPF, effective renal plasma flow; RPF, renal plasma flow; Hct, haematocrit; RBF, renal blood flow.

\section{Discussion}

This study confirms that RBF estimation based on ${ }^{82} \mathrm{Rb}$ PET/CT using AA as the IF in a 1-tissue compartment model is feasible, as previously indicated by Tahari et al. (21). Additionally, our results support the use of the AA-VOI in a single FOV as an alternative IF to the LVBP; the low intra-assay coefficients of variation are acceptable with excellent inter-observer reliability, thus allowing estimated RBF to be determined using a single FOV assessment of the kidneys in their entirety. To our knowledge, this is the first study to assess method precision and determine intraassay variation and inter-observer variability for RBF estimates with ${ }^{82} \mathrm{Rb} \mathrm{PET} / \mathrm{CT}$. However as 
1 discussed below, we believe the renal clearance of ${ }^{82} \mathrm{Rb}\left(\mathrm{K}_{1}\right)$, to represent ERPF, rather than direct

2 estimation of RBF.

\section{${ }^{82} \mathrm{Rb}$ as renal perfusion tracer}

4 There are many advantages to using ${ }^{82} \mathrm{Rb} \mathrm{PET} / \mathrm{CT}$ for measurement of renal perfusion: it is non-

5 invasive and does not require blood sampling or urine collection, making the procedure less

6 burdensome for patients; it allows for single kidney blood flow estimation and is readily available

7 from ${ }^{82} \mathrm{Sr} /{ }^{82} \mathrm{Rb}$ generators which are already in-situ at sites routinely using ${ }^{82} \mathrm{Rb}$ for assessment of

8 myocardial blood flow, thus making it cost effective. In comparison, the "ideal tracer" - ${ }^{15} \mathrm{O}-\mathrm{water}-$

9 can be utilized only in centres with on-site cyclotron access (31). The combination of a short ${ }^{82} \mathrm{Rb}$

10 half-life of 75 seconds and short acquisition time allows for repeated scans of the same subject

11 within a short timeframe, presenting unique opportunities to examine acute effects of differing

12 drugs on renal perfusion. For example, ${ }^{82} \mathrm{Rb}$ PET/CT may be especially suitable for use in cross-

13 over studies exploring interventional effects.

14 No absolute contraindications exist to the use of ${ }^{82} \mathrm{Rb}$, thus patients suffering from all stages of AKI

15 and $\mathrm{CKD}$ can undergo the examination without risk of deterioration of renal function.

16 Since renal ${ }^{82} \mathrm{Rb}$ accumulation exceeds myocardial ${ }^{82} \mathrm{Rb}$ accumulation, half the tracer dose of

17 cardiac studies is sufficient to perform good quality renal imaging, resulting in a low effective

18 radiation dose $(\sim 1 \mathrm{mSv})$ for a single scan of the kidneys in their entirety, including the AA for use

19 as IF. Additionally, for modern digital scanners with high sensitivities, even lower tracer doses may

20 be sufficient to perform the examination.

\section{Input functions and necessary data correction}

22 Pharmacokinetic modelling requires an IF, where sampling of peripheral arterial blood to produce

23 an arterial TAC is the gold standard method for obtaining an accurate estimation. However, the

24 short half-life of ${ }^{82} \mathrm{Rb}$ necessitates an alternative to the arterial sampling derived input curve. This 
1 can be achieved using image derived curves based on e.g. PET/CT scanning, where LVBP and AA

2 are examples of IDIFs. Accurate quantitative IDIF estimation is dependent on many parameters,

3 relating to both the individual PET/CT scanner and reconstruction parameters used for imaging, the

4 geometry, size and placement of analysis VOIs with respect to structural organs-of-interest

5 boundaries, the ratio of neighbouring activity concentrations, as well as requiring calibration of the

$6 \quad{ }^{82} \mathrm{Rb}$-tracer injector system and imaging scanner with associated dose calibrators; the 3 primary

7 sources of quantitation error being scanner count efficiency, PVE and spill-over. As a minimum, an

8 understanding of what corrections are, and are not, automatically included in an individual scanner-

9 systems software, is necessary to verify correct method implementation and data analysis locally. If

10 one can ensure the relative contributions from PVE and spill-over are negligible in all VOIs used to

11 obtain organ specific TAC data, the assumption that any global scanner-specific error will cancel

12 out in the kinetic modelling should be adequate and allow for evaluation of activity concentrations

13 without need for cross-calibration of all systems. However, if it is not possible to ensure negligible

14 PVE and spill-over effects in one or more of the VOIs, then the relative activity concentrations

15 defining the TACs will not be correct with respect to each another and will result in an erred kinetic

16 analysis of renal flow. One method to reduce PVE is to define VOIs as $1 \mathrm{~cm}^{3}$ volume spheres

17 centered on the highest activity voxel in the organ of interest and measure peak-activity

18 concentrations. However, placement of smaller VOIs is variable and observer dependent, especially

19 in highly inhomogeneous (biological) activity distributions. As such, it can be advantageous to use

20 mean values to define the activity measurements. Additionally, if the maximum voxel count lies in

21 proximity to an organ boundary, PVE and spill-over will not be reduced and will still have to be

22 accounted for in the data analysis.

23 We obtained uncorrected IDIFs from TACs based on VOIs placed in both LVBP and AA in the

24 dynamic PET images. Use of IDIFs based on large-size vascular structures, combined with the high 25 resolution of modern PET scanners, reduces PVE in activity measurement $(32,33)$. Additional

26 investigation of measured activity accuracy as a function of distance from structural boundaries 
1 (specific to our scanner and reconstruction method), using a phantom containing known ${ }^{82} \mathrm{Rb}$

2 activity concentrations in geometrical structures simulating the volumes, shapes and sizes of the

3 LVBP and AA (unpublished data), showed that to ensure negligible PVE and spillover effects when

4 defining a VOI, its placement needs to be a minimum of $15 \mathrm{~mm}$ from organ boundaries; ie. a

5 minimum of 3-5 voxels distance, dependent on the choice of imaging matrix. In the smaller AA

6 structure, even though there is very little background to give unwanted spill-in, this criteria was not

7 met, indicating that PVE is present and requires correction. On the other hand, the large LVBP

8 volume indicates that PVE is reduced. However, due to significant uptake of ${ }^{82} \mathrm{Rb}$ activity in the left

9 ventricular wall and the use of hot-contouring producing VOIs with, at most, 1-2 voxel distances

10 from the myocardium, the LVBP also required correction for PVE and spill-over. Both corrections

11 were performed based on the method of Katoh et. al, (28) using equations 1 and 2, with the

12 necessary correction factors experimentally determined from phantom measurements. Additionally,

13 a global calibration of our scanner's count efficiency in a large $\left(>100 \mathrm{~cm}^{3}\right)$ homogeneous volume

14 was made, to provide kidney VOI data correction. Here, PVE and spill-over are negligible, but

15 count efficiency is not automatically corrected by the Siemens scanner software. Based on these

16 arguments, 3 differing values for "organ specific" $\beta$-values were required to ensure the correct

17 relative relationships between the corrected organ-TAC data; one cannot assume a single, global

18 scanner and reconstruction dependent correction factor, will cancel out in subsequent kinetic

19 analysis, unless the employed VOI definition protocol ensures independence from PVE and spill-

20 over in all organs.

21 Comparison of our IFs with those of the first (and to date only published) human renal ${ }^{82} \mathrm{Rb}$ PET/CT study by Tahari et al. (21), show both similarities and differences. In both studies, the uncorrected activity in the AA is observed to be lower than LVBP activity. As there is no known metabolism of ${ }^{82} \mathrm{Rb}$ in its passage through the aorta, it is assumed that the activity concentrations in the left ventricle and the aortic lumen are equal and as such, observed measurement differences will be caused by any scanner and image reconstruction quantification inaccuracies, as discussed above. 
1 Tahari et. al. (21) assessed the effect to arise from PVE and performed the correction using a simple

2 scaling of their measured AA activity to match the observed maximum LVBP activity. It is unclear

3 whether LVBP activity in (21) was corrected for PVE and spill-over. Our more systematic

4 approach, in which calibrated phantom measurements determined the recovery coefficients $\beta$

5 necessary to correct VOI specific activity measurements for a given organ geometry, gave $\beta$-values

6 of $0.71,0.612$ and 0.643 respectively for LV, AA and kidney TAC corrections, where the numerical

7 value for $\beta$ does not differentiate between the relative contributions from PVE, spill-over or count

8 efficiency, but provides a "global" factor accounting for all contributions. Application of these

9 organ-specific $\beta$-values increased the measured peak values for both LVBP and AA TACs (Fig. 6

10 and Fig. 7) resulting in the corrected-AA IFs being scaled to match the corrected LVBP IFs (Fig. 8);

11 supporting the assumption that activity concentrations in the left ventricle and aortic lumen are

12 equal. This is in agreement with the IFs shown in Tahari's study. The main difference is that our $\mathrm{K}_{1}$

13 values obtained from AA IFs, differ from their AA $\mathrm{K}_{1}$ derived values, due to our correction of

14 kidney-TACs for system counting efficiency.

15 Using the $\beta$-corrected TAC data, we find for both AA and LVBP, the intra-assay coefficients of

16 variation are acceptably low, indicating that ${ }^{82} \mathrm{Rb}$ PET/CT is a precise method for evaluation of $\mathrm{K}_{1}$,

17 hence allowing for determination of changes in $\mathrm{K}_{1}$. Additionally, the inter-observer variability

18 assessment supports the use of AA as IF as a robust image-derived method for determining renal

19 perfusion, with excellent reliability demonstrated for both kidneys using AA, compared to good to

20 excellent reliability using LVBP.

\section{Renal clearance - measurement of $K_{1}$, estimation of ERPF and RBF}

22 High renal ${ }^{82} \mathrm{Rb}$ uptake and accumulation were confirmed. To avoid errors in uptake estimation 23 caused by regional differences, it is important to measure uptake in the entire kidney. In our study,

2413 out of 18 completing subjects (72\%) showed both LVBP and the entire kidneys in FOV-A, such 25 that 5 analyses were performed on truncated kidneys. In the article by Tahari et al. (21), only 3 out 
1 of 8 subjects (38\%) had the LVBP and kidneys in the same FOV (corresponding to FOV-A) and 5

2 out of 8 subjects had the LVBP and kidneys in separate acquisitions. As a global quality control, we

3 found no significant difference between $\mathrm{K}_{1}$ values derived from AA activity curves in FOV-A and

4 those in FOV-B, supporting the assumption that in the studied population with healthy, lesion-free

5 kidneys, quantitation obtained from truncated images of the kidney tissue is representative of values

6 which would be obtained from imaging the kidneys in their entirety. This may not, however, be

7 universally true. Since blood flow substantially differs between the renal cortex and outer and inner

8 medulla, the extent of kidney tissue included in the analysis VOI may affect values of measured

9 blood flow. Even though the poor quality of the low-dose CT, used for AC-correction only, did not

10 allow discrimination of the cortex and medulla in our kidney VOIs, due to the good quality images

11 and high renal uptake observed in ${ }^{82} \mathrm{Rb}$ PET/CT imaging, this differential flow measurement is

12 potentially possible using CT contrast enhancement or even PET/MR from which to define the

13 kidney VOIs. Using $1 \mathrm{~cm}^{3}$ equivalent to $1 \mathrm{~g}$ of tissue, individual kidney volumes can be

14 approximated from the volumes encompassed by the kidney VOIs, allowing conversion of $\mathrm{K}_{1}$

15 values $\left(\mathrm{ml} / \mathrm{min} / \mathrm{cm}^{3}\right)$ to total flow values for both kidneys ( $\left.\mathrm{ml} / \mathrm{min}\right)$. Total flow values are

16 summarized in Table 5.

17 Our clearance values (expressed as ERPF), are observed to be low when compared to previously

18 published mean values for RBF: 1100-1500 $\mathrm{ml} / \mathrm{min}(34,35)$; however, they are quite similar, if

19 somewhat at the high end, to previously published mean values for ERPF with values 345-700

$20 \mathrm{ml} / \mathrm{min} / 1.73 \mathrm{~m}^{2}(34,36,37)$. This is suggests ${ }^{82} \mathrm{Rb}$ PET/CT may actually be estimating RPF and not

$21 \mathrm{RBF}$ as is the current understanding.

22 Whether we measure estimated RBF or ERPF with ${ }^{82} \mathrm{Rb}$ depends on the distribution of the tracer

23 between plasma and erythrocytes in whole blood. Early studies of potassium permeability showed a

24 very slow exchange of radioactive potassium and rubidium between plasma and erythrocytes

25 amounting to $1.8-2.1 \%$ per hour and even less over 8 minutes of study $(25,26)$. Hence, most ${ }^{82} \mathrm{Rb}$ is

26 present in the plasma during renal uptake studies, implying the measured renal uptake values 
1 represent estimated RPF after correction for extraction, if EF is close to unity. Assuming our data

2 represents RPF, estimated RBF can be calculated by correcting with the haematocrit value which is

3 easily measured; the results of which are presented in Table 5.

4 For canines, EF is estimated to be $0.89(0.80-0.95)$ (19), but to our knowledge remains to be

5 determined in humans due to difficulty in calibrating and measuring blood activity for ${ }^{82} \mathrm{Rb}$.

6 However, if we assume the extraction values to be similar for humans, we find an average total

7 estimated RBF value normalized to BSA of $1494 \pm 221 \mathrm{ml} / \mathrm{min} / 1.73 \mathrm{~m}^{2}$, which lies at the upper end

8 of the expected general range for RBF in healthy subjects. Since our study population consisted of a

9 highly homogeneous group of young, healthy subjects, our estimation of a high ERPF, and

10 consequently a high RBF is to be expected; our results being consistent with 2 early studies of

11 similar population groups, published ca $1960(34,35)$, where mean RBF values in the range 1100 -

$121500 \mathrm{ml} / \mathrm{min}$, were calculated based on measurement of PAH-clearance and using conversion for

13 extraction fraction and haematocrit. Specifically, our mean and range of estimated RBF values are

14 fully consistent with the published range of individual RBF values $(1150-2350 \mathrm{ml} / \mathrm{min})$ in the

15 study by Brodwall et. al (35).

\section{Study strengths and limitations}

17 The major strengths of this study are a combination of the randomized cross-over design, the

18 standardization of pre-scan conditions (fluid intake, exercise level, duration of fasting period), and

19 the consecutive acquisition of the four ${ }^{82} \mathrm{Rb}$ PET/CT scans over a short 45 -minute period; enabling

20 optimal evaluation of intra-assay coefficients of variation and hereby precision. Additionally, the

21 use of measured recovery coefficients provides reliable numerical correction of the IFs which are

22 specific to our PET/CT scanner, imaging reconstruction method and choice of VOI definitions.

23 The homogeneous study population consisted of healthy adults, providing estimated RBF

24 measurements uninfluenced by age and medical therapy. However, this is also a potential bias as it

25 is not certain results from this study can be directly applied to a population of elderly subjects, nor 
1 to subjects suffering from hypertension or renal disease; additional feasibility studies may be

2 needed for these populations. Additionally, before ${ }^{82} \mathrm{Rb}$ PET/CT can be implemented for clinical

3 estimated RBF determination, further evaluation is required of day-to-day variation as well as the

4 quantitative accuracy of the method.

5 A technical limitation is the use of the automatic injection system used to provide the bolus

6 administration of activity; in practice a short infusion of ${ }^{82} \mathrm{Rb}$ is administered, which, depending on

7 the age of the ${ }^{82} \mathrm{Sr} /{ }^{82} \mathrm{Rb}$ generator can have a duration between 20 - 40 seconds, and as such does

8 not represent a true bolus injection which should ideally be administered within 10 seconds. For this

9 reason, low activity may be present in the kidneys even before the activity in the blood pool has

10 peaked, as seen in Fig. 8.

11 A major limitation of the study is the assumption that the extraction fraction and blood volume

12 fraction, as derived from animal experiments, are valid for calculation of RBF in humans from

13 measurement of ERPF. Due to a general lack of published literature for ${ }^{82} \mathrm{Rb}$ renal flow

14 measurement, with early research based nearly exclusively on animal studies (1990s and earlier)

15 and the first (and as far as we are aware, only) human investigation performed and published by

16 Tahari et. al. in 2014, no solid data is available for humans, such that animal based values are the

17 best, and only, data available to us.

18 Another limitation of this study is that it does not provide comparison to a reference method; the

19 accuracy of ${ }^{82} \mathrm{Rb}$ PET/CT for RBF estimation cannot be evaluated. Based on the available literature

20 during study design, the assumption was that the method provides a measurement of RBF for which

21 an appropriate reference method would be comparison with ${ }^{15} \mathrm{O}$-water studies (31). However, in

22 light of the results presented here, if we are in fact measuring plasma flow and not, as originally

23 assumed RBF, then additional reference methods become available, such as $\mathrm{PAH} / \mathrm{OIH}$-clearance

24 methods $(34,36,37)$. In fact, comparison of ${ }^{82} \mathrm{Rb}$ measured $\mathrm{K}_{1}$ flow values, with a reference 
1 method for ERPF evaluation, could help answer the question regarding which quantity is actually

2 being measured in ${ }^{82} \mathrm{Rb} \mathrm{PET} / \mathrm{CT}$ studies.

\section{Conclusion}

4 The results presented in this study, for a population of young, healthy subjects, support the use of an

5 AA IDIF in the 1-tissue compartment model as an alternative to LVBP; it is sufficient to determine

6 estimated RBF using a single FOV including AA and kidneys in their entirety using a single

7 dynamic ${ }^{82} \mathrm{Rb}$ PET/CT scan. Accurate quantification of the AA derived IDIF requires PVE

8 corrections, and eventual count efficiency calibration, relevant for the imaging scanner and

9 reconstruction method employed. Use of AA gave rise to an acceptably low intra-assay coefficient

10 of variation $(\sim 5 \%)$ and good to excellent inter-observer reliability.

11 Our data suggests that the actual flow values measured by ${ }^{82} \mathrm{Rb}$ most likely represent ERPF rather

12 than RBF which is essential for the correct interpretation of future perfusion studies using

$13 \quad{ }^{82} \mathrm{Rb}$. Abbreviations

14 AA: abdominal aorta; AKI: Acute kidney injury; ALAT: Alanine aminotransferase; BMI: Body

15 mass index; BSA: Body surface area; CI: Confidence interval; CKD: Chronic kidney disease; CT:

16 Computed tomography; EF: Extraction fraction; ERPF: Effective renal plasma flow; FOV: Field of

17 view; ICC: Intra-class correlation coefficient; IDIF: Image-derived input function; IF: Input

18 function; LVBP: Left ventricular blood pool; MBq: Megabecquerel; mCi: Millicurie; mSv:

19 MilliSievert; ${ }^{15} \mathrm{O}$ water: oxygen-15 labeled water; PET: Positron emission tomography; p.i.: Post

20 injection; PVE: Partial volume effect; ${ }^{82} \mathrm{Rb}$ : Rubidium-82; RBF: Renal blood flow; ${ }^{82} \mathrm{Sr}$ : Strontium-

21 82; SD: Standard deviation; TAC: time activity curve; VOI: volume of interest. 


\section{Declarations}

3 Ethics approval and consent to participate

4 The study was approved by the Regional Scientific Ethics Committee (journal number: 1-10-72-

5 175-16), the Danish Medicines Agency (EudraCT-number: 2016-004080-39), the Danish Data

6 Protection Agency, and was conducted in agreement with the Declaration of Helsinki 2013.

7 Informed written consent was obtained from all participants.

8 Consent for publication

9 Informed consent was obtained from all participants regarding publishing of data.

10 Availability of data and materials

11 The datasets and trial protocol (Danish) are available from the corresponding author on reasonable 12 request.

\section{Competing interest}

14 The authors declare that they have no competing interest.

\section{Funding}

16 The study was supported by The Axel Muusfeld Foundation and The Research Foundation of the

17 Central Denmark Region.

18

19 Authors' contributions

20 Study concept \& design: all authors; Method implementation, creation \& validation of analysis

21 tools: JT, CF; Additional phantom studies, interpretation of quantification results and application in 22 data analysis: CF, JT; Data acquisition: SSL; Data analysis: SSL, JT; Data interpretation: SSL, JT, 23 CF; drafting of manuscript: SSL; Critical revision: SSL, JT, CF, JNB; Critical revision revised 24 manuscript: CF, JT, SSL; Approval of final manuscript: all authors. 
1 The authors thank all the Medical Laboratory Technologists involved in the practical performance

2 of this study protocol: Malene Skov Hansen \& Mette Emtkjær Mølgaard (PET/CT scanning) and

3 Henriette Vorup Simonsen \& Kirsten Nygaard (blood sample analysis).

4

5

\section{References}

$7 \quad 1$. Parekh DJ, Weinberg JM, Ercole B, Torkko KC, Hilton W, Bennett M, et al.

8 Tolerance of the human kidney to isolated controlled ischemia. J Am Soc Nephrol. 2013;24(3):506-

917.

102 Saotome T, Ishikawa K, May CN, Birchall IE, Bellomo R. The impact of

11 experimental hypoperfusion on subsequent kidney function. Intensive Care Med. 2010;36(3):533-

1240.

13 3. Prowle JR, Molan MP, Hornsey E, Bellomo R. Measurement of renal blood flow by

14 phase-contrast magnetic resonance imaging during septic acute kidney injury: a pilot investigation.

15 Crit Care Med. 2012;40(6):1768-76.

16 4. Kreimeier U, Hammersen F, Ruiz-Morales M, Yang Z, Messmer K. Redistribution of

17 intraorgan blood flow in acute, hyperdynamic porcine endotoxemia. Eur Surg Res. 1991;23(2):85-

1899.

$195 . \quad$ Di Giantomasso D, May CN, Bellomo R. Vital organ blood flow during

20 hyperdynamic sepsis. Chest. 2003;124(3):1053-9.

216 Khatir DS, Pedersen M, Jespersen B, Buus NH. Evaluation of Renal Blood Flow and

22 Oxygenation in CKD Using Magnetic Resonance Imaging. American Journal of Kidney Diseases :

23 The Official Journal of the National Kidney Foundation. 2015;66(3):402-11. 
2 Flow in Chronic Kidney Disease Using Arterial Spin Labeling Perfusion Magnetic Resonance

3 Imaging. Kidney international reports. 2017;2(1):36-43.

48 Goldring W, Chasis H, Ranges HA, Smith HW. Effective Renal Blood Flow in

5 Subjects with Essential Hypertension. The Journal of clinical investigation. 1941;20(6):637-53.

$69 . \quad$ Reubi FC, Weidmann P, Hodler J, Cottier PT. Changes in renal function in essential

7 hypertension. The American Journal of Medicine. 1978;64(4):556-63.

8 10. Hollenberg NK, Epstein M, Basch RI, Merrill JP. "No man's land" of the renal

9 vasculature. An arteriographic and hemodynamic assessment of the interlobar and arcuate arteries

10 in essential and accelerated hypertension. Am J Med. 1969;47(6):845-54.

11 11. van Hooft IM, Grobbee DE, Derkx FH, de Leeuw PW, Schalekamp MA, Hofman A.

12 Renal hemodynamics and the renin-angiotensin-aldosterone system in normotensive subjects with

13 hypertensive and normotensive parents. The New England journal of medicine. 1991;324(19):1305-

1411.

15 12. Uneda S, Fujishima S, Fujiki Y, Tochikubo O, Oda H, Asahina S, et al. Renal

16 haemodynamics and the renin-angiotensin system in adolescents genetically predisposed to

17 essential hypertension. J Hypertens Suppl. 1984;2(3):S437-9.

18 13. Battilana C, Zhang HP, Olshen RA, Wexler L, Myers BD. PAH extraction and

19 estimation of plasma flow in diseased human kidneys. Am J Physiol. 1991;261(4 Pt 2):F726-33.

20 14. Stadalnik RC, Vogel JM, Jansholt AL, Krohn KA, Matolo NM, Lagunas-Solar MC, et

21 al. Renal clearance and extraction parameters of ortho-iodohippurate (I-123) compared with OIH(I-

22 131) and PAH. Journal of nuclear medicine : official publication, Society of Nuclear Medicine.

$23 \quad 1980 ; 21(2): 168-70$.

24 15. Russell CD, Thorstad B, Yester MV, Stutzman M, Baker T, Dubovsky EV.

25 Comparison of technetium-99m MAG3 with iodine-131 hippuran by a simultaneous dual channel 26 technique. J Nucl Med. 1988;29(7):1189-93. 
116. Kalantarinia K, Belcik JT, Patrie JT, Wei K. Real-time measurement of renal blood

2 flow in healthy subjects using contrast-enhanced ultrasound. Am J Physiol Renal Physiol.

$3 \quad$ 2009;297(4):F1129-34.

4 Z17. Ziadi MC, Dekemp RA, Williams KA, Guo A, Chow BJ, Renaud JM, et al. Impaired 5 myocardial flow reserve on rubidium-82 positron emission tomography imaging predicts adverse 6 outcomes in patients assessed for myocardial ischemia. J Am Coll Cardiol. 2011;58(7):740-8.

$7 \quad$ 18. El Fakhri G, Kardan A, Sitek A, Dorbala S, Abi-Hatem N, Lahoud Y, et al.

8 Reproducibility and accuracy of quantitative myocardial blood flow assessment with (82)Rb PET:

9 comparison with (13)N-ammonia PET. Journal of nuclear medicine : official publication, Society of

10 Nuclear Medicine. 2009;50(7):1062-71.

11 Mullani NA, Ekas RD, Marani S, Kim EE, Gould KL. Feasibility of measuring first 12 pass extraction and flow with rubidium-82 in the kidneys. American Journal of Physiologic 13 Imaging. 1990;5(4):133-40.

14 20. Senthamizhchelvan S, Bravo PE, Esaias C, Lodge MA, Merrill J, Hobbs RF, et al. 15 Human biodistribution and radiation dosimetry of $82 \mathrm{Rb}$. Journal of nuclear medicine : official 16 publication, Society of Nuclear Medicine. 2010;51(10):1592-9.

17 21. Tahari AK, Bravo PE, Rahmim A, Bengel FM, Szabo Z. Initial human experience 18 with Rubidium-82 renal PET/CT imaging. Journal of medical imaging and radiation oncology. $19 \quad 2014 ; 58(1): 25-31$.

Weinberg IN, Huang SC, Hoffman EJ, Araujo L, Nienaber C, Grover-McKay M, et al.

21 Validation of PET-acquired input functions for cardiac studies. Journal of nuclear medicine :

22 official publication, Society of Nuclear Medicine. 1988;29(2):241-7.

23 23. Germino M, Ropchan J, Mulnix T, Fontaine K, Nabulsi N, Ackah E, et al.

24 Quantification of myocardial blood flow with (82)Rb: Validation with (15)O-water using time-of25 flight and point-spread-function modeling. EJNMMI research. 2016;6(1):68-016-0215-6. Epub 262016 Aug 1. 
2 effect of captopril on renal blood flow in renal artery stenosis assessed by positron tomography with

3 rubidium-82. Hypertension. 1988;11(3):217-22.

$425 . \quad$ Sheppard CW, Martin WR. Cation exchange between cells and plasma of mammalian

5 blood; methods and application to potassium exchange in human blood. J Gen Physiol.

$6 \quad 1950 ; 33(6): 703-22$.

$726 . \quad$ Love WD, Burch GE. A comparison of potassium 42, rubidium 86, and cesium 134 as

8 tracers of potassium in the study of cation metabolism of human erythrocytes in vitro. J Lab Clin

9 Med. 1953;41(3):351-62.

10 27. Hsu B. PET tracers and techniques for measuring myocardial blood flow in patients

11 with coronary artery disease. J Biomed Res. 2013;27(6):452-9.

12 28. Katoh C, Yoshinaga K, Klein R, Kasai K, Tomiyama Y, Manabe O, et al.

13 Quantification of regional myocardial blood flow estimation with three-dimensional dynamic

14 rubidium-82 PET and modified spillover correction model. Journal of nuclear cardiology : official

15 publication of the American Society of Nuclear Cardiology. 2012;19(4):763-74.

$1629 . \quad$ Effros RM, Lowenstein J, Baldwin DS, Chinard FP. Vascular and extravascular

17 volumes of the kidney of man. Circulation research. 1967;20(2):162-73.

$18 \quad 30 . \quad$ Koo TK. A Guideline of Selecting and Reporting Intraclass Correlation Coefficients

19 for Reliability Research. Journal of chiropractic medicine. 2016;15(2):155-63.

20 31. Prior JO, Allenbach G, Valenta I, Kosinski M, Burger C, Verdun FR, et al.

21 Quantification of myocardial blood flow with $82 \mathrm{Rb}$ positron emission tomography: clinical

22 validation with $15 \mathrm{O}$-water. European journal of nuclear medicine and molecular imaging.

$23 \quad 2012 ; 39(6): 1037-47$.

$24 \quad 32$.

de Geus-Oei LF, Visser EP, Krabbe PF, van Hoorn BA, Koenders EB, Willemsen AT,

et al. Comparison of image-derived and arterial input functions for estimating the rate of glucose metabolism in therapy-monitoring 18F-FDG PET studies. J Nucl Med. 2006;47(6):945-9. 
2 Image-derived input functions for determination of MRGlu in cardiac (18)F-FDG PET scans. J

$3 \quad$ Nucl Med. 2001;42(11):1622-9.

4 34. Bergstrom J, Bucht H, Ek J, Josephson B, Sundell H, Werko L. The renal extraction

5 of para-aminohippurate in normal persons and in patients with diseased kidneys. Scandinavian

6 Journal of Clinical and Laboratory Investigation. 1959;11:361-75.

$735 . \quad$ Brodwall EK. Renal Extraction of Pah in Normal Individuals. Scandinavian Journal of

$8 \quad$ Clinical and Laboratory Investigation. 1964;16:1-5.

9 36. Prenen JA, de Klerk JM, van het Schip AD, van Rijk PP. Technetium-99m-MAG3

10 versus iodine-123-OIH: renal clearance and distribution volume as measured by a constant infusion

11 technique. Journal of nuclear medicine : official publication, Society of Nuclear Medicine.

$12 \quad 1991 ; 32(11): 2057-60$.

13 37. Tietze IN, Pedersen EB. Renal haemodynamic changes, renal tubular function, sodium

14 and water homeostatic hormones in patients with chronic glomerulonephritis and in healthy humans

15 after intravenous infusion of amino acids. Nephrology, dialysis, transplantation : official publication 16 of the European Dialysis and Transplant Association - European Renal Association. 1994;9(5):49917504.

\section{Figure legends}

Fig. 1 Study design

Fig. 2 Anatomical contents within the $22 \mathrm{~cm}$ axial FOV for an example study image (subject 2): a) FOV-A: bed position includes LVBP, AA and kidneys (body length and kidney size determine whether the kidneys can be seen in their entirety within the FOV); b) FOV-B bed position includes AA and kidneys in their entirety 
2 Fig. 3 1-tissue compartment model used for estimation of RBF. $\mathrm{K}_{1}$ is the rate constant for ${ }^{82} \mathrm{Rb}$ uptake in the kidneys from the vascular space, whereas $\mathrm{k}_{2}$ is the rate constant for release of ${ }^{82} \mathrm{Rb}$ back into the blood. No discernible tracer activity was observed via urinary excretion (21)

Fig. 4 VOIs were drawn in a) myocardium, b) left ventricular blood pool, c \& d) abdominal aorta and aortic background (orange - aorta; purple - background) and e) contouring the kidneys (green - right; cerise - left)

$9 \quad$ Fig. 5 Participant flow in the study

11 Fig. 6 Representative time activity curves from the left ventricular blood pool and the myocardium from one of the 12 study subjects

14 Fig. 7 Representative time activity curves from the abdominal aorta and the aortic background from one of the study subjects.

17 Fig. 8 Typical time activity curves from the left ventricular blood pool, abdominal aorta, and the kidneys from one of the study subjects. The observed presence of low activity in the kidneys before the activity in the blood pool has peaked

19 is due to the automatic injection system for delivery of ${ }^{82} \mathrm{Rb}$ providing an infusion of the radioisotope over a $20-40$ second period, which does not constitute a true bolus injection.

23 Fig. 9 Typical example of a) coronal and b) transaxial PET/CT images of kidneys during maximal ${ }^{82} \mathrm{Rb}$ uptake from one of the study subjects. Shown example is for FOV-B 
Figures

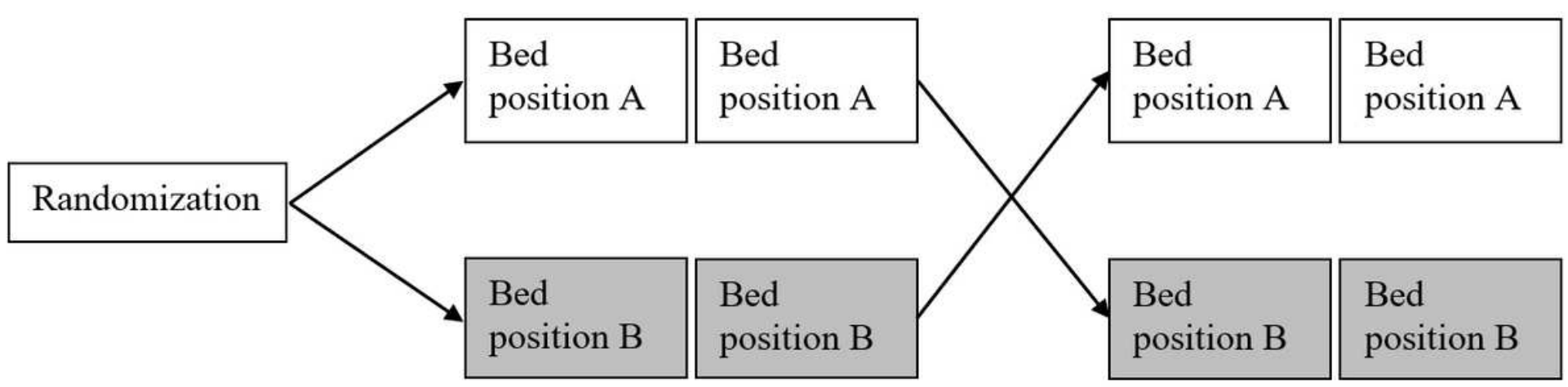

$45 \mathrm{~min}$

Figure 1

Study design

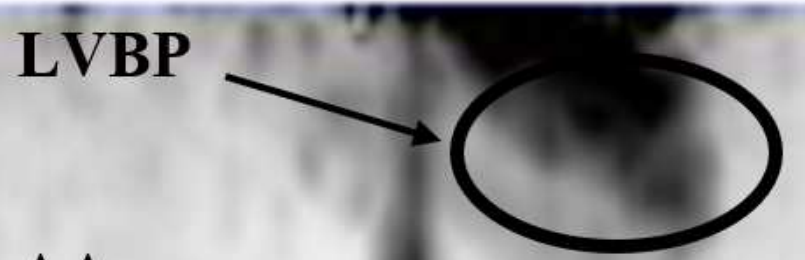

AA

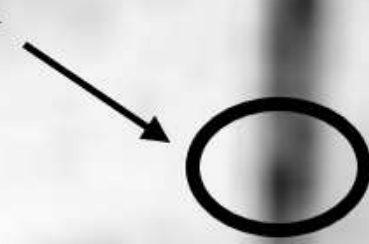

LK

RK

a

b

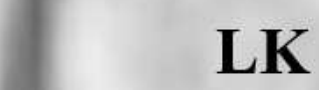
.

AA

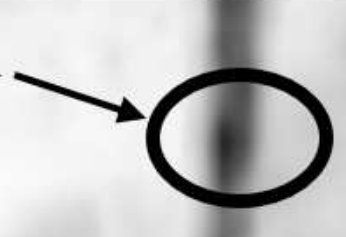

LK

\section{RK}




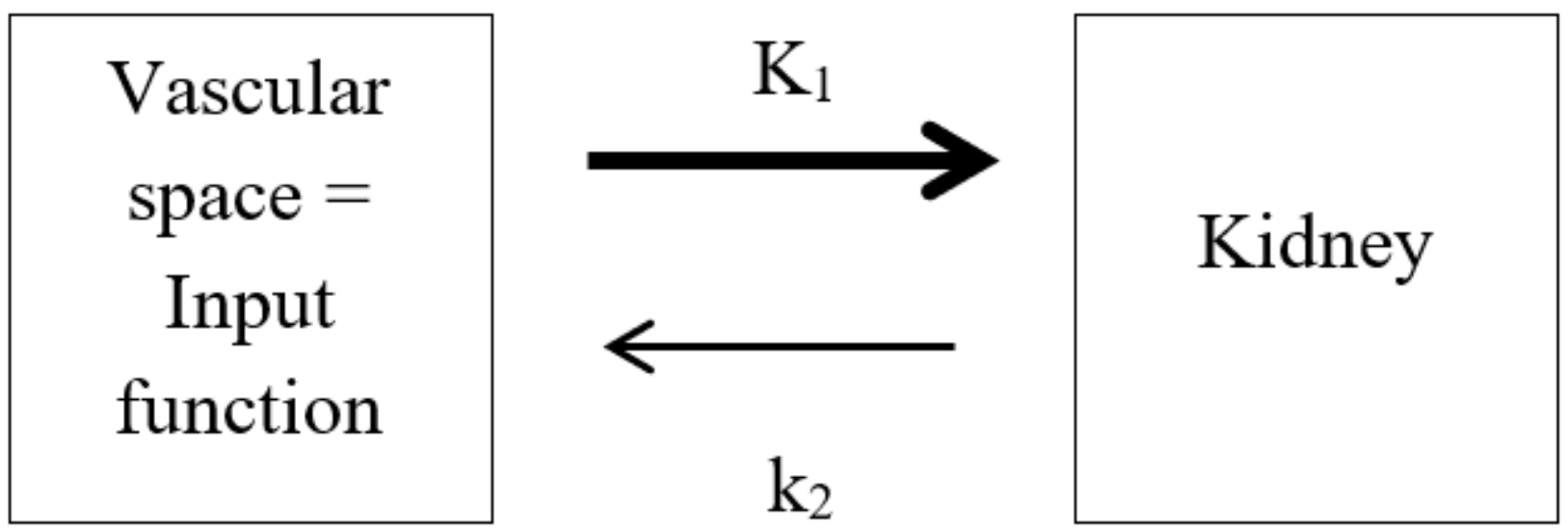

Figure 3

1-tissue compartment model used for estimation of RBF. K1 is the rate constant for $82 \mathrm{Rb}$ uptake in the kidneys from the vascular space, whereas $\mathrm{k} 2$ is the rate constant for release of $82 \mathrm{Rb}$ back into the blood. No discernible tracer activity was observed via urinary excretion (21) 
$\mathbf{a}$

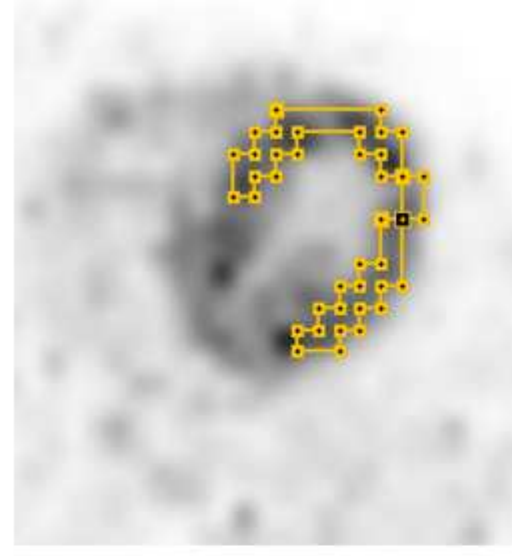

\begin{abstract}
b
\end{abstract}

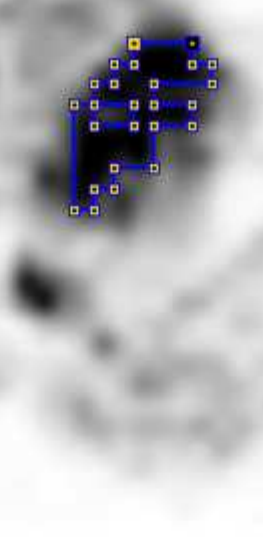

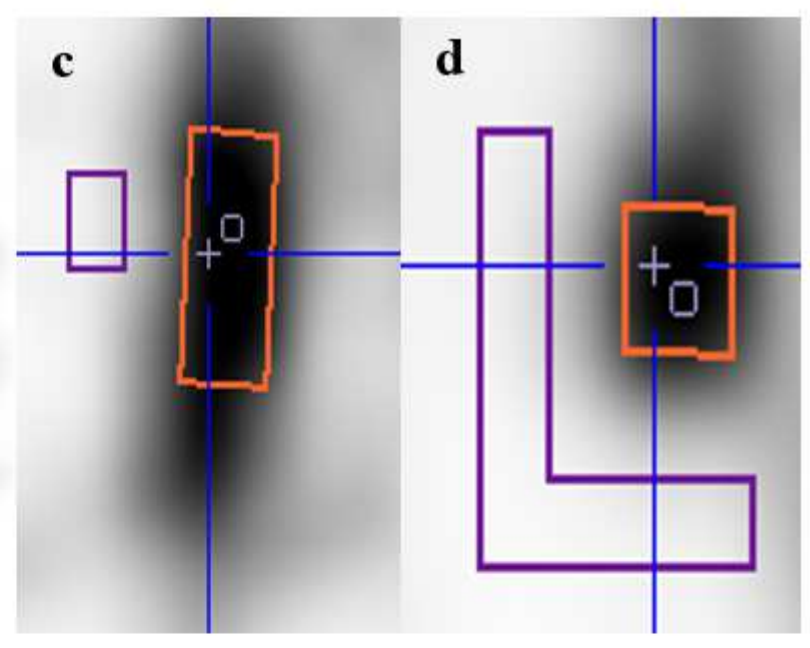
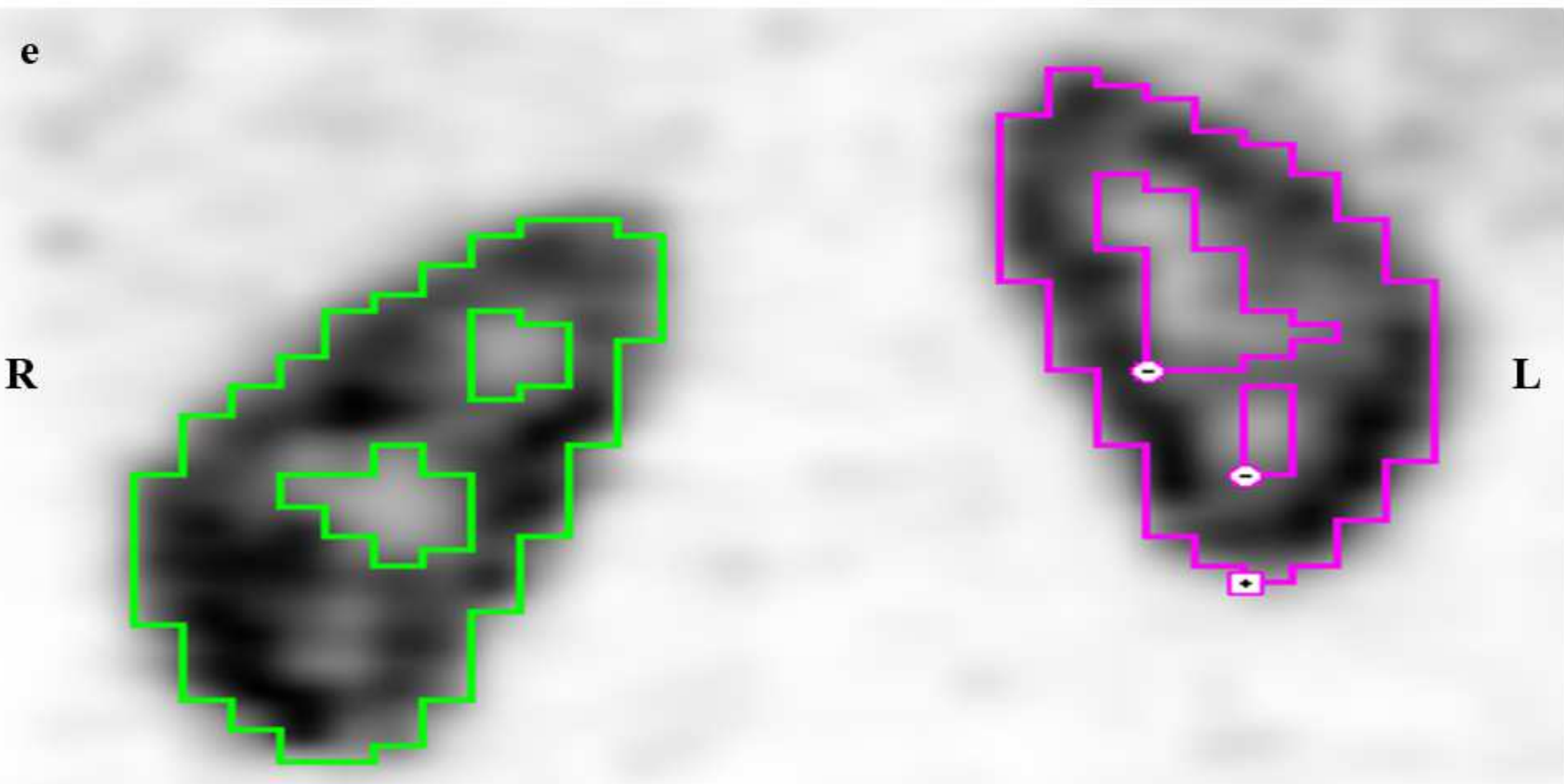

\section{Figure 4}

VOIs were drawn in a) myocardium, b) left ventricular blood pool, c \& d) abdominal aorta and aortic background (orange - aorta; purple - background) and e) contouring the kidneys (green - right; cerise left) 


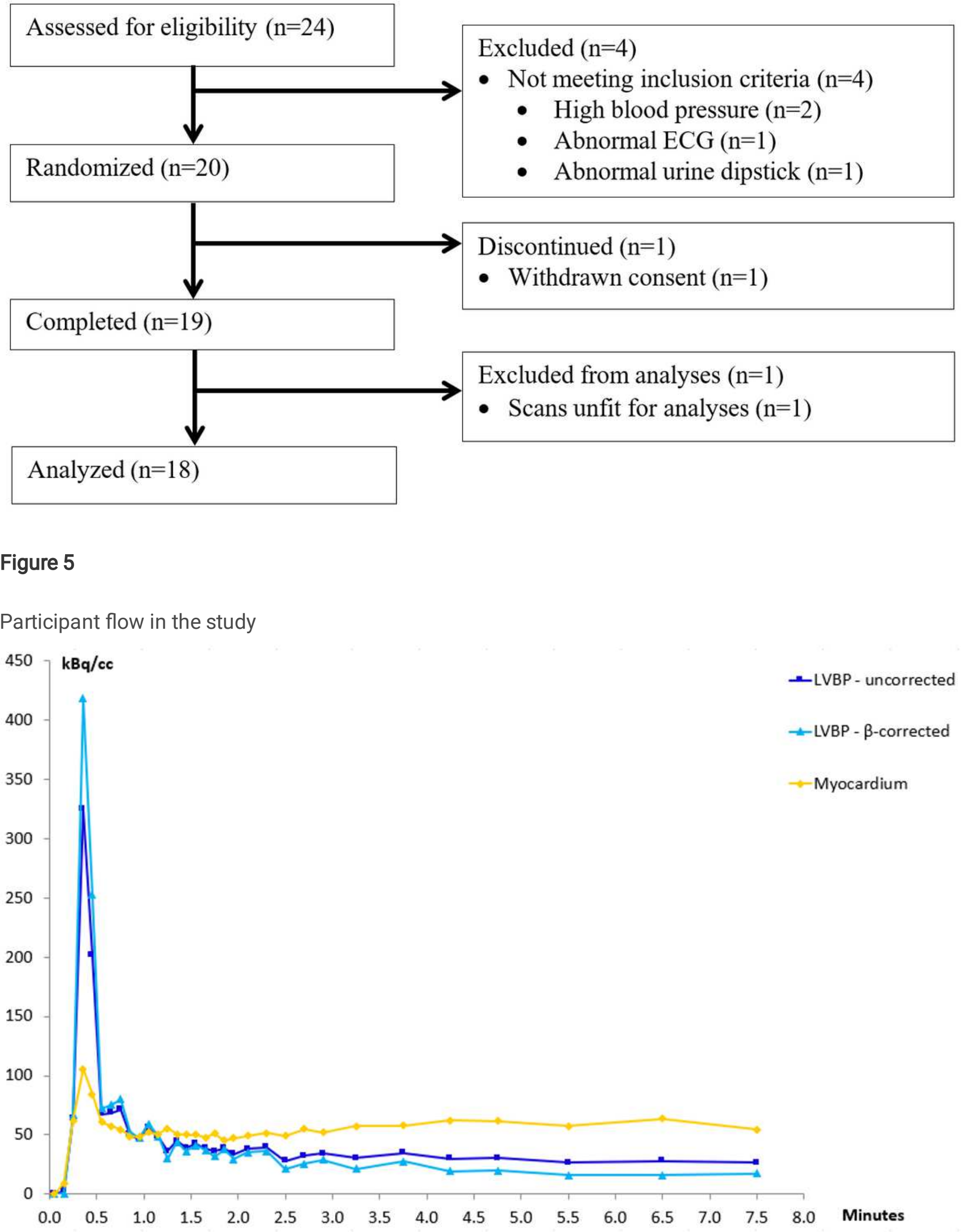


Figure 6

Representative time activity curves from the left ventricular blood pool and the myocardium from one of the study subjects

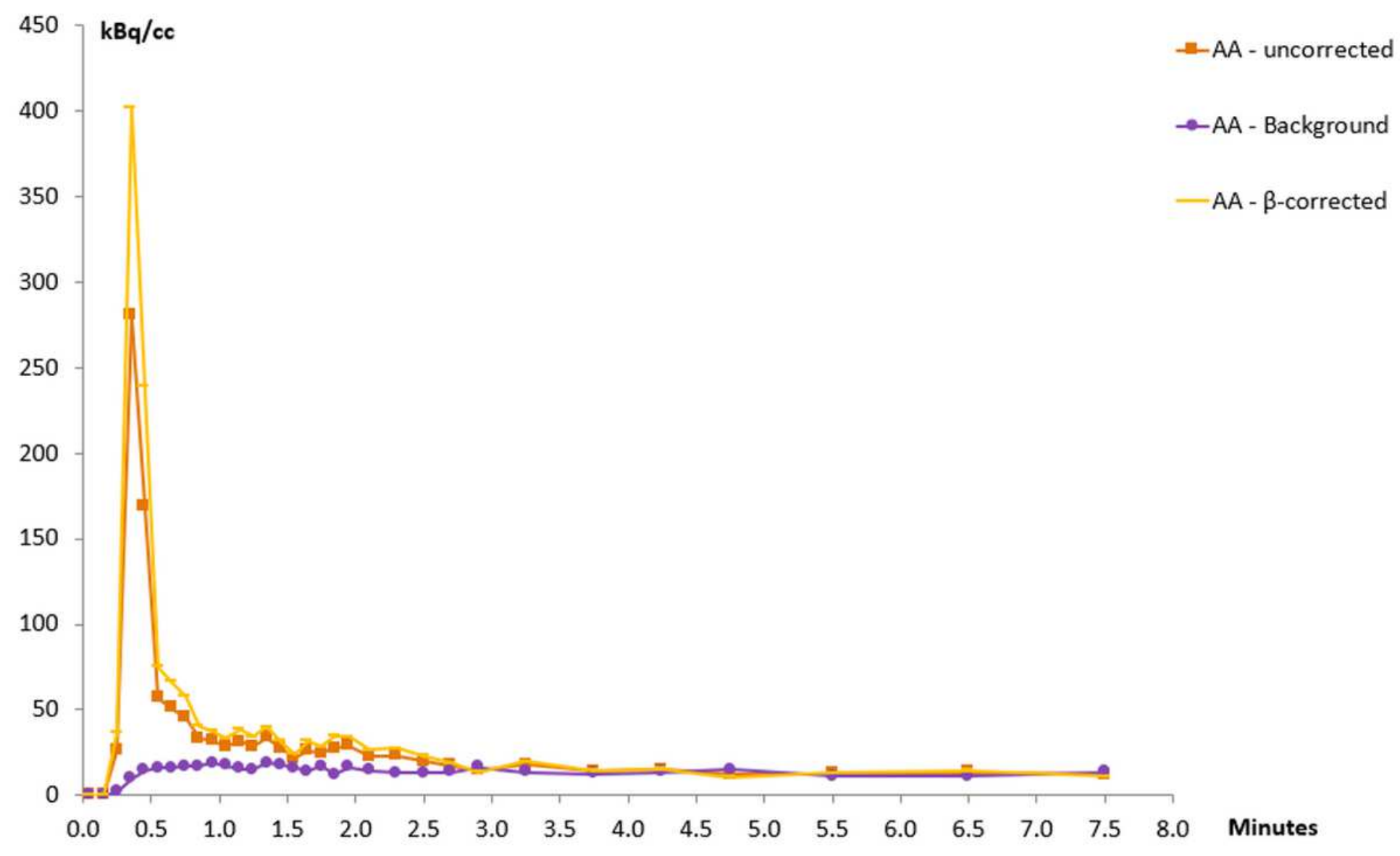

Figure 7

Representative time activity curves from the abdominal aorta and the aortic background from one of the study subjects. 


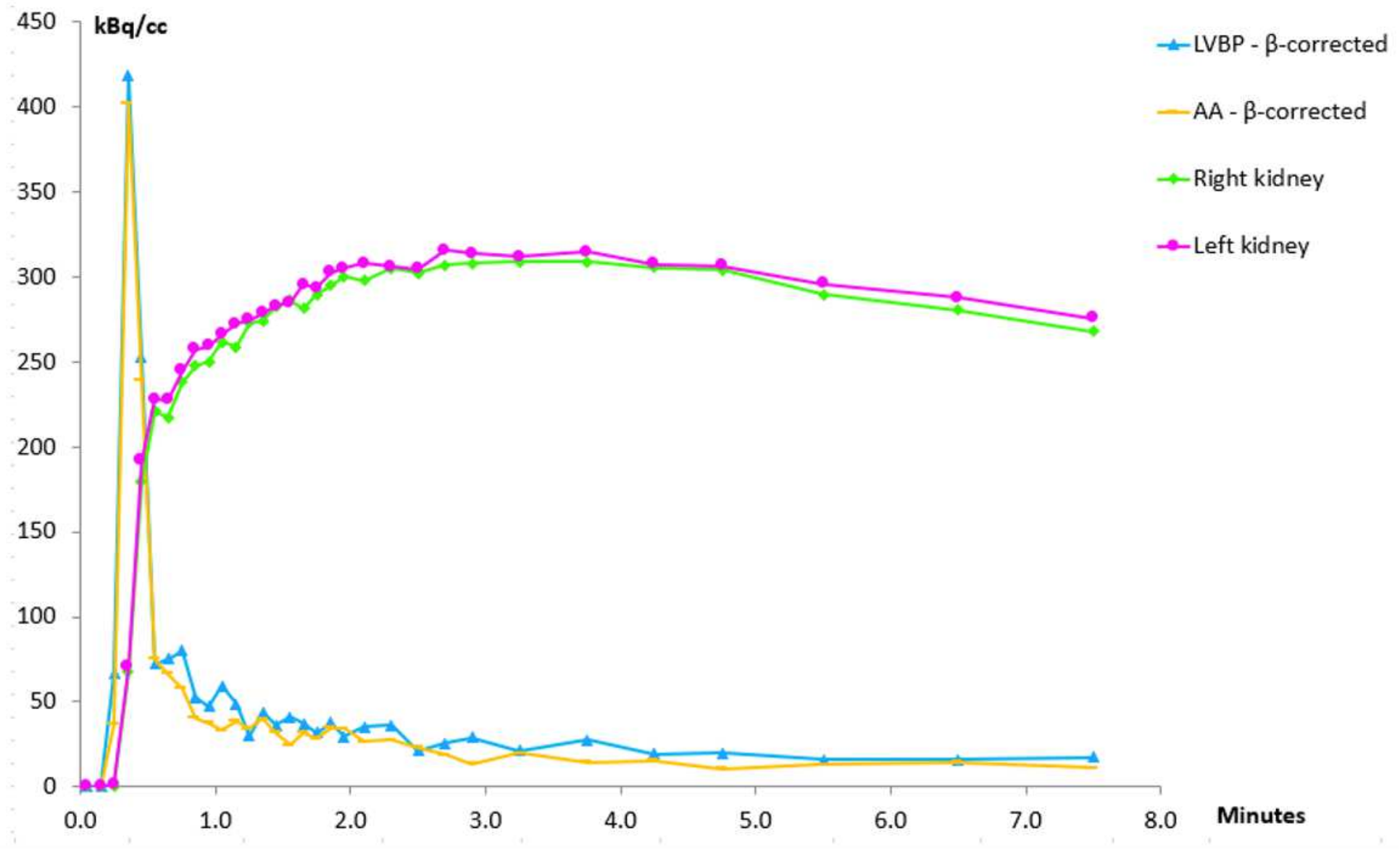

Figure 8

Typical time activity curves from the left ventricular blood pool, abdominal aorta, and the kidneys from one of the study subjects. The observed presence of low activity in the kidneys before the activity in the blood pool has peaked is due to the automatic injection system for delivery of $82 \mathrm{Rb}$ providing an infusion of the radioisotope over a 20-40 second period, which does not constitute a true bolus injection. 


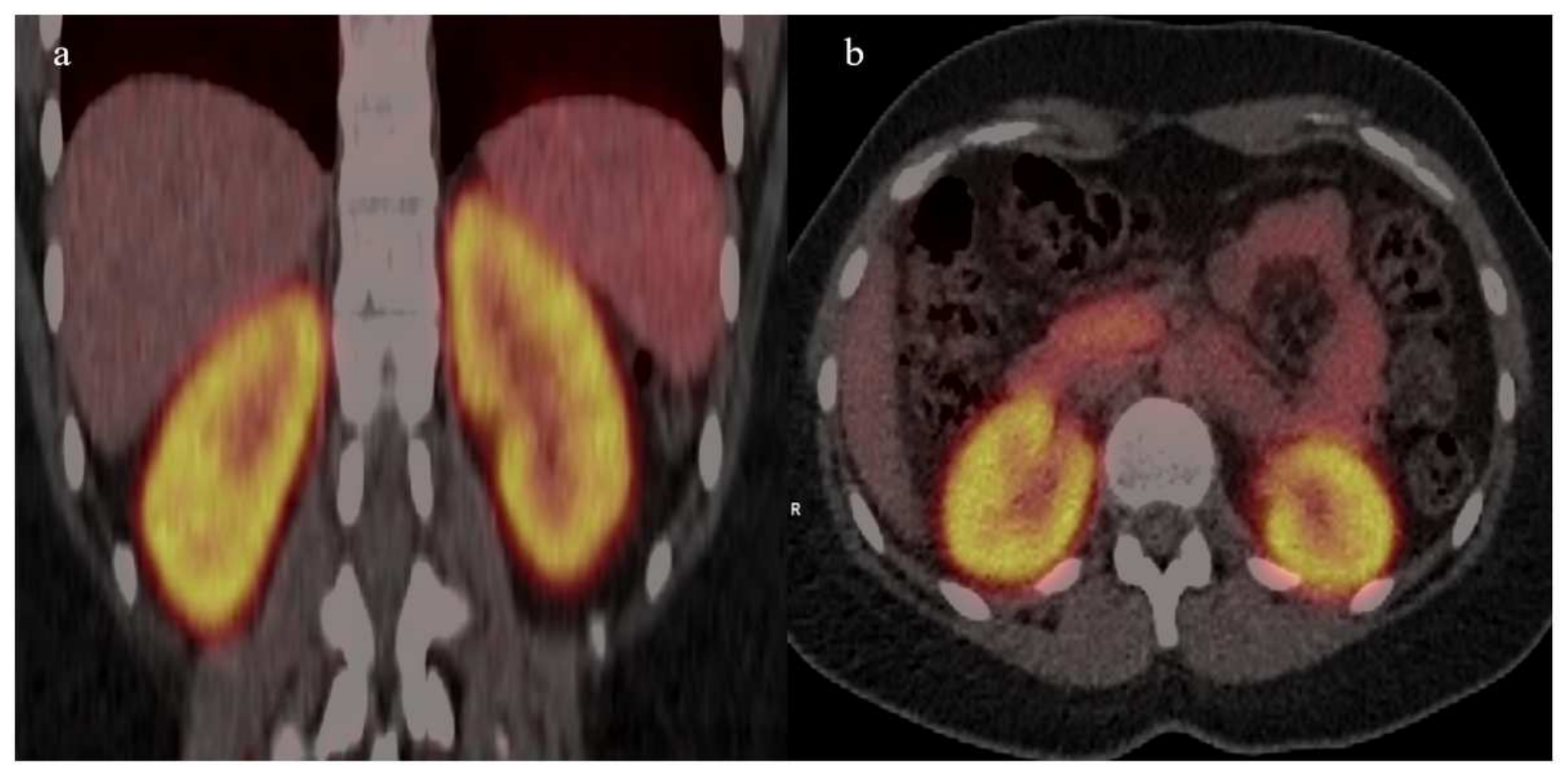

\section{Figure 9}

Typical example of a) coronal and b) transaxial PET/CT images of kidneys during maximal 82Rb uptake from one of the study subjects. Shown example is for FOV-B 